\title{
Mean-Square Stability of Milstein Methods for Stochastic Pantograph Equations
}

\author{
Feiyan Xiao, ${ }^{1,2}$ Tingting Qin, ${ }^{1}$ and Chengjian Zhang ${ }^{1}$ \\ ${ }^{1}$ School of Mathematics and Statistics, Huazhong University of Science and Technology, Wuhan 430074, China \\ ${ }^{2}$ School of Mathematics and Statistics, Guangxi Normal University, Guilin 541004, China \\ Correspondence should be addressed to Tingting Qin; tingtingqin@hust.edu.cn
}

Received 1 June 2013; Revised 22 September 2013; Accepted 27 September 2013

Academic Editor: Guo-Cheng Wu

Copyright (c) 2013 Feiyan Xiao et al. This is an open access article distributed under the Creative Commons Attribution License, which permits unrestricted use, distribution, and reproduction in any medium, provided the original work is properly cited.

\begin{abstract}
This paper deals with nonlinear stochastic pantograph equations. For solving the equations, a class of extended Milstein methods are suggested. A mean-square stability criterion for this type of equations is presented. It is proved that under the suitable conditions the Milstein methods preserve the mean-square stability. Numerical examples further illustrate the obtained theoretical results.
\end{abstract}

\section{Introduction}

Stochastic delay differential equations (SDDEs) are often used to model some problems with aftereffect in many scientific fields such as physics, biology, mechanics, finance, and control theory. Generally speaking, it is hard to obtain the analytical solutions of SDDEs. Hence, recently, many researchers began to study their numerical solutions, and hence, some significant results have been achieved.

The stability analysis plays an important role in construction of excellent numerical algorithms for SDDEs. Hence, it has received wide attention of researchers. The early related results can be found in Mao [1,2], Baker and Buckwar [3], Buckwar [4, 5], Küchler and Platen [6], and the references therein. More recently, for the linear SDDEs, Cao et al. [7], Liu et al. [8], and Wang and Zhang [9] studied mean-square stability (MS-stability) of Euler-Maruyama, semi-implicit EulerMaruyama, and Milstein methods, respectively. Taking use of the Halanay inequality, Baker and Buckwar [10] extended the MS-stability analysis of Euler-Maruyama methods to nonlinear SDDEs. Moreover, Wang and Zhang [11] also dealt with nonlinear MS-stability of Milstein methods.

We note that the above numerical stability investigations were mainly devoted to the case of constant delay. Although the deterministic delay differential equations with variable delays have been widely studied (see, e.g., $[12,13]$ and the references therein), the case of variable delay of SDDEs was rarely concerned. Fan and Liu [14] first studied linear stochastic pantograph equations and gave MS-stability criteria of semi-implicit Euler methods. Also, by taking use of the analytical and discrete Razumikhin theorems, they dealt with $\alpha$-moment stability of linear stochastic pantograph equations and their semi-implicit Euler method (cf. [15]). Recently, Xiao et al. $[16,17]$ gave sufficient MS-stability conditions of backward Euler method and semi-implicit Euler method with variable stepsize for linear stochastic pantograph differential equations. In the present paper, we will investigate the MSstability of nonlinear stochastic pantograph equations and their Milstein methods. Some criteria for MS-stability of the analytical and numerical solutions will be derived. Numerical experiments will be used to illustrate the obtained theoretical results.

\section{MS-Stability of the Analytical Solutions}

Let $(\Omega, \mathscr{A}, P)$ be a complete probability space with a filtration $\left(\mathscr{A}_{t}\right)_{t \geq 0}$, which is right-continuous and satisfies that each $\mathscr{A}_{t}(t \geq 0)$ contains all $P$-null sets in $\mathscr{A}$, and $w$ is a one-dimensional Brownian motion defined on the probability space. Moreover, we introduce the following notations:

$$
\left.|\cdot|:|A|=\sqrt{\operatorname{trace}\left(A^{T} A\right)} \text { (the trace norm of matrix } A\right) \text {; }
$$


$L^{p}\left(\Omega, R^{d}\right)$ : the family of $R^{d}$-value random variable $x$ with $E|x|^{p}<\infty$;

$\mathscr{L}^{p}\left([a, b], R^{d}\right)$ : the family of $R^{d}$-value $\mathscr{A}_{t}$-adapted

$$
\begin{aligned}
& \text { processes }\{x(t)\}_{a \leq t \leq b} \\
& \text { with } \int_{a}^{b}|x(t)|^{p} d t<\infty \quad \text { a.s.; }
\end{aligned}
$$

$\mathscr{M}^{p}\left([a, b], R^{d}\right):$ the family of processes $\{x(t)\}_{t \geq 0}$

$$
\begin{aligned}
& \in \mathscr{L}^{p}\left([a, b], R^{d}\right) \\
& \text { with } E \int_{a}^{b}|x(t)|^{p} d t<\infty
\end{aligned}
$$

$\mathscr{L}^{p}\left(R_{+}, R^{d}\right):$ the family of processes $\{x(t)\}_{t \geq 0}$

$$
\begin{array}{r}
\text { with }\{x(t)\}_{0 \leq t \leq T} \in \mathscr{L}^{p}\left([0, T], R^{d}\right) \\
\forall T>0 ;
\end{array}
$$

$\mathscr{M}^{p}\left(R_{+}, R^{d}\right)$ : the family of processes $\{x(t)\}_{t \geq 0}$

$$
\begin{array}{r}
\text { with }\{x(t)\}_{0 \leq t \leq T} \in \mathscr{M}^{p}\left([0, T], R^{d}\right) \\
\forall T>0 .
\end{array}
$$

Consider the following nonlinear stochastic pantograph equations:

$$
\begin{gathered}
d x(t)=f(t, x(t), x(p t)) d t \\
+g(t, x(t), x(p t)) d w(t), \quad t>0, \\
x(0)=\xi
\end{gathered}
$$

where $x(t)$ is a $R^{d}$-value random process, $p \in(0,1)$ denotes a given constant, $f: R_{+} \times R^{d} \times R^{d} \rightarrow R^{d}$ and $g: R_{+} \times R^{d} \times R^{d} \rightarrow$ $R^{d}$ are two given Borel-measurable functions, $\xi$ is an $\mathscr{A}_{0^{-}}$ measurable $R^{d}$-value random variable, and $\xi \in L^{2}\left(\Omega, R^{d}\right)$. Throughout this paper, we always assume that (2) has a unique solution $x(t) \in \mathscr{M}^{2}\left(R_{+}, R^{d}\right)$.

Definition 1. The solution of (2) is said to be MS-stable if

$$
\lim _{t \rightarrow+\infty} E|x(t)|^{2}=0
$$

Theorem 2. Assume that there exist constants $\alpha>0, \beta \geq 0$, and $\gamma \geq 0$ such that

$$
\begin{aligned}
& x^{T} f(t, x, u) \leq-\alpha|x|^{2}+\beta|u|^{2}, \quad \forall x, u \in R^{d}, \\
& |g(t, x, u)|^{2} \leq \gamma\left(|x|^{2}+|u|^{2}\right), \quad \forall x, u \in R^{d} .
\end{aligned}
$$

Then, the solution of (2) is MS-stable whenever

$$
\gamma-2 \alpha+\frac{\gamma+2 \beta}{p}<0
$$

Proof. By the Itô formula (cf. [1]), we have

$$
\begin{aligned}
& d|x(t)|^{2} \\
& =\left[2 x^{T}(t) f(t, x(t), x(p t))+|g(t, x(t), x(p t))|^{2}\right] d t \\
& \quad+2 x^{T}(t) g(t, x(t), x(p t)) d w(t) .
\end{aligned}
$$

Integrating from 0 to $t$ on both sides of the equality (7) and then taking expectation yield that

$$
\begin{array}{r}
E|x(t)|^{2}=E|\xi|^{2}+E \int_{0}^{t}\left[2 x^{T}(s) f(s, x(s), x(p s))\right. \\
\left.+|g(s, x(s), x(p s))|^{2}\right] d s \\
+E \int_{0}^{t} 2 x^{T}(s) g(s, x(s), x(p s)) d w(s) .
\end{array}
$$

Since $x(t) \in \mathscr{M}^{2}\left(R_{+}, R^{d}\right)$, we further have

$$
\begin{aligned}
E|x(t)|^{2}=E|\xi|^{2}+E \int_{0}^{t}[ & 2 x^{T}(s) f(s, x(s), x(p s)) \\
& \left.+|g(s, x(s), x(p s))|^{2}\right] d s .
\end{aligned}
$$

Applying the conditions (4) and (5) to (9), it follows that

$$
\begin{aligned}
E|x(t)|^{2} \leq & E|\xi|^{2}+(\gamma-2 \alpha) E \int_{0}^{t}|x(s)|^{2} d s \\
& +(\gamma+2 \beta) E \int_{0}^{t}|x(p s)|^{2} d s \\
\leq & E|\xi|^{2}+\left(\gamma-2 \alpha+\frac{\gamma+2 \beta}{p}\right) E \int_{0}^{t}|x(s)|^{2} d s
\end{aligned}
$$

which gives

$$
-\left(\gamma-2 \alpha+\frac{\gamma+2 \beta}{p}\right) E \int_{0}^{t}|x(s)|^{2} d s \leq E|\xi|^{2}, \quad \forall t>0 .
$$

This, together with (6), implies $\lim _{t \rightarrow \infty} E|x(t)|^{2}=0$. Therefore, the theorem is proven.

\section{MS-Stability of the Numerical Solutions}

For the stability analysis, we introduce the following notational conventions:

$$
\begin{gathered}
g_{1}^{\prime}(t, x, u)=\frac{\partial g(t, x, u)}{\partial x}, \quad g_{2}^{\prime}(t, x, u)=\frac{\partial g(t, x, u)}{\partial u}, \\
I_{1}=\int_{t_{n}}^{t_{n+1}} \int_{t_{n}}^{s} d w(r) d w(s)=\frac{\left(\Delta w_{n}\right)^{2}-h}{2} \\
I_{2}=\int_{t_{n}}^{t_{n+1}} \int_{t_{n}}^{s} d w(p r) d w(s),
\end{gathered}
$$

where $\Delta w_{n}:=\int_{t_{n}}^{t_{n+1}} d w(s)=w\left(t_{n+1}\right)-w\left(t_{n}\right)$, denoting independent $N(0, h)$-distributed Gaussian random variables. 
Moreover, on space $R^{d}$, we define an inner product $\langle\cdot, \cdot\rangle$ and the corresponding induced norm $|\cdot|$ as follows:

$$
\langle U, V\rangle=\sum_{i=1}^{d} u_{i} v_{i}, \quad|U|=\sqrt{\sum_{i=1}^{d} u_{i}^{2}}
$$

where $U=\left(u_{1}, u_{2}, \ldots, u_{d}\right)^{T}, V=\left(v_{1}, v_{2}, \ldots, v_{d}\right)^{T} \in R^{d}$.

Applying the Milstein method to (2) derives the following numerical scheme:

$$
\begin{gathered}
x_{n+1}=x_{n}+h f\left(t_{n}, x_{n}, \bar{x}_{n}\right)+g\left(t_{n}, x_{n}, \bar{x}_{n}\right) \Delta w_{n} \\
+g_{1}^{\prime}\left(t_{n}, x_{n}, \bar{x}_{n}\right) g\left(t_{n}, x_{n}, \bar{x}_{n}\right) I_{1} \\
+g_{2}^{\prime}\left(t_{n}, x_{n}, \bar{x}_{n}\right) g\left(t_{n}, \bar{x}_{n}, \hat{x}_{n}\right) I_{2}, \quad n \geq 0, \\
x_{0}=\xi,
\end{gathered}
$$

where $h>0$ is the computational stepsize, $\Delta w_{n}=w\left(t_{n+1}\right)-$ $w\left(t_{n}\right)$, and $x_{n}, \bar{x}_{n}$, and $\widehat{x}_{n}$ are approximations to $x\left(t_{n}\right), x\left(p t_{n}\right)$, and $x\left(p^{2} t_{n}\right)$, respectively. When set

$$
p t_{n}=\left(n-v_{n}\right) h+\delta_{n} h, \quad p^{2} t_{n}=\left(n-\bar{v}_{n}\right) h+\bar{\delta}_{n} h,
$$

where $v_{n}, \bar{\nu}_{n} \in \mathbb{N}$ and $\delta_{n}, \bar{\delta}_{n} \in[0,1)$, the approximations of $x\left(p t_{n}\right)$ and $x\left(p^{2} t_{n}\right)$ can be defined as follows:

$$
\begin{gathered}
\bar{x}_{n}=\delta_{n} x_{n-v_{n}+1}+\left(1-\delta_{n}\right) x_{n-v_{n}}, \\
\widehat{x}_{n}=\bar{\delta}_{n} x_{n-\bar{v}_{n}+1}+\left(1-\bar{\delta}_{n}\right) x_{n-\bar{v}_{n}}, \quad n \geq 0 .
\end{gathered}
$$

In this way, an extended Milstein method, composed by (14) and (16), is obtained.

Definition 3. An extended Milstein method (14)-(16) is said to be MS-stable if there exists an $h_{0}>0$ such that

$$
\lim _{n \rightarrow+\infty} E\left|x_{n}\right|^{2}=0, \quad h \in\left(0, h_{0}\right] .
$$

Lemma 4. The Itô-type double integrals $I_{1}, I_{2}$ have the following properties:

$$
E\left[I_{1}\right]=E\left[I_{2}\right]=0, \quad E\left|I_{1}\right|^{2}=\frac{h^{2}}{2}, \quad E\left|I_{2}\right|^{2}=\frac{p h^{2}}{2} .
$$

Proof. The equalities $E\left[I_{1}\right]=E\left[I_{2}\right]=0$ can be derived directly from the properties of martingales. Moreover, by the equality $I_{1}=\left[(\Delta w)^{2}-h\right] / 2$, we have

$$
E\left|I_{1}\right|^{2}=\frac{1}{4} E\left[\left(\Delta w_{n}\right)^{2}-h\right]^{2}=\frac{h^{2}}{2} .
$$

Also, it follows from the properties of Itô integral that

$$
\begin{aligned}
E\left|I_{2}\right|^{2} & =E\left[\int_{t_{n}}^{t_{n+1}} \int_{t_{n}}^{s} d w(p r) d w(s)\right]^{2} \\
& =\int_{t_{n}}^{t_{n+1}} E\left[\int_{t_{n}}^{s} d w(p r)\right]^{2} d s \\
& =\int_{t_{n}}^{t_{n+1}} \int_{t_{n}}^{s} d(p r) d s=\frac{p h^{2}}{2} .
\end{aligned}
$$

This completes the proof.
Let $q=1-p$. Then, we have the following lemma.

Lemma 5. Assume that there exist positive integers $r, v_{i}$ and $\delta_{i} \in[0,1)$ such that

$$
r \leq \frac{1}{q}<r+1, \quad i q=v_{i}-\delta_{i}, \quad i=0,1,2, \ldots
$$

Then, the sequence $\left\{v_{i}\right\}$ is monoincreasing and has at most $r+1$ equal components.

Proof. It follows from iq $=v_{i}-\delta_{i}$ that

$$
v_{i+1}+\delta_{i}=v_{i}+\delta_{i+1}+q, \quad i=0,1,2, \ldots
$$

Let $\lfloor\cdot\rfloor$ denote the integer part of a real number. Then, by $q, \delta_{i} \in[0,1)$ and $v_{i} \in \mathbb{N}$, we have for all $i$ that

$$
\left\lfloor v_{i+1}+\delta_{i}\right\rfloor=v_{i+1}, \quad\left\lfloor v_{i}+\delta_{i+1}+q\right\rfloor \geq v_{i} .
$$

Hence, it holds that

$$
v_{i+1} \geq v_{i}, \quad i=0,1,2, \ldots,
$$

This shows that the sequence $\left\{v_{i}\right\}$ is monoincreasing.

For proving the second part of this lemma, we use reduction to absurdity. If the sequence $\left\{v_{i}\right\}$ has $r+2$ components which satisfy that

$$
v_{i_{0}}=v_{i_{1}}=\cdots=v_{i_{r+1}}, \quad \text { where } 0 \leq i_{0}<i_{1}<\cdots<i_{r+1} \text {, }
$$

then, by $v_{i_{r+1}}-v_{i_{0}}=0, \delta_{i_{r+1}} \in[0,1)$, and $q>1 /(r+1)$, we have

$$
\delta_{i_{0}}=\delta_{i_{r+1}}+\left(i_{r+1}-i_{0}\right) q \geq \frac{i_{r+1}-i_{0}}{r+1} \geq 1 .
$$

This is contrary to $\delta_{i_{0}} \in[0,1)$. Hence, Lemma 5 is proven.

With the above lemmas, the main result can be stated as follows.

Theorem 6. Assume that the conditions (4) and (5) hold and that there exist constants $\kappa, M$, and $N \geq 0$ such that

$$
\begin{gathered}
|f(t, x, u)|^{2} \leq \kappa\left(|x|^{2}+|u|^{2}\right), \quad x, u \in R^{d}, \\
\left|g_{1}^{\prime}(t, x, u)\right| \leq M, \quad\left|g_{2}^{\prime}(t, x, u)\right| \leq N, \quad x, u \in R^{d} .
\end{gathered}
$$

Then, the extended Milstein method (14)-(16) is MS-stable whenever

$$
c_{1}+2 c_{2}(r+1)+2 c_{3}(r+1)<0,
$$

where

$$
\begin{gathered}
c_{1}=-2\left(\alpha-2 \gamma-M^{2} \gamma\right), \\
c_{2}=2\left(\beta+2 \gamma+M^{2} \gamma+N^{2} \gamma p\right), \quad c_{3}=2 N^{2} \gamma p .
\end{gathered}
$$


Proof. By (14), we have

$$
\begin{aligned}
& \left|x_{n+1}\right|^{2} \leq\left|x_{n}\right|^{2} \\
& +2\left\langle x_{n}, h f\left(t_{n}, x_{n}, \bar{x}_{n}\right)+g\left(t_{n}, x_{n}, \bar{x}_{n}\right) \Delta w_{n}\right. \\
& +g_{1}^{\prime}\left(t_{n}, x_{n}, \bar{x}_{n}\right) g\left(t_{n}, x_{n}, \bar{x}_{n}\right) I_{1} \\
& \left.+g_{2}^{\prime}\left(t_{n}, x_{n}, \bar{x}_{n}\right) g\left(t_{n}, \bar{x}_{n}, \widehat{x}_{n}\right) I_{2}\right\rangle \\
& +\mid h f\left(t_{n}, x_{n}, \bar{x}_{n}\right)+g\left(t_{n}, x_{n}, \bar{x}_{n}\right) \Delta w_{n} \\
& +g_{1}^{\prime}\left(t_{n}, x_{n}, \bar{x}_{n}\right) g\left(t_{n}, x_{n}, \bar{x}_{n}\right) I_{1} \\
& +\left.g_{2}^{\prime}\left(t_{n}, x_{n}, \bar{x}_{n}\right) g\left(t_{n}, \bar{x}_{n}, \widehat{x}_{n}\right) I_{2}\right|^{2} \\
& \leq\left|x_{n}\right|^{2}+2 h x_{n}^{T} f\left(t_{n}, x_{n}, \bar{x}_{n}\right) \\
& +2 x_{n}^{T}\left[g\left(t_{n}, x_{n}, \bar{x}_{n}\right) \Delta w_{n}\right] \\
& +2 x_{n}^{T}\left[g_{1}^{\prime}\left(t_{n}, x_{n}, \bar{x}_{n}\right) g\left(t_{n}, x_{n}, \bar{x}_{n}\right) I_{1}\right] \\
& +2 x_{n}^{T}\left[g_{2}^{\prime}\left(t_{n}, x_{n}, \bar{x}_{n}\right) g\left(t_{n}, \bar{x}_{n}, \widehat{x}_{n}\right) I_{2}\right] \\
& +4 h^{2}\left|f\left(t_{n}, x_{n}, \bar{x}_{n}\right)\right|^{2}+4\left|g\left(t_{n}, x_{n}, \bar{x}_{n}\right)\right|^{2}\left|\Delta w_{n}\right|^{2} \\
& +4\left|g_{1}^{\prime}\left(t_{n}, x_{n}, \bar{x}_{n}\right)\right|^{2}\left|g\left(t_{n}, x_{n}, \bar{x}_{n}\right)\right|^{2}\left|I_{1}\right|^{2} \\
& +4\left|g_{2}^{\prime}\left(t_{n}, x_{n}, \bar{x}_{n}\right)\right|^{2}\left|g\left(t_{n}, \bar{x}_{n}, \widehat{x}_{n}\right)\right|^{2}\left|I_{2}\right|^{2} \text {. }
\end{aligned}
$$

Using conditions (4) and (27) generates

$$
\begin{gathered}
E\left[x_{n}^{T} f\left(t_{n}, x_{n}, \bar{x}_{n}\right)\right] \leq-\alpha E\left|x_{n}\right|^{2}+\beta E\left|\bar{x}_{n}\right|^{2}, \\
E\left|f\left(t_{n}, x_{n}, \bar{x}_{n}\right)\right|^{2} \leq \kappa\left(E\left|x_{n}\right|^{2}+E\left|\bar{x}_{n}\right|^{2}\right),
\end{gathered}
$$

respectively. Moreover, the $\mathscr{A}_{t_{n}}$-measurability implies that

$$
\begin{aligned}
& E\left[x_{n}^{T}\left[g\left(t_{n}, x_{n}, \bar{x}_{n}\right) \Delta w_{n}\right]\right] \\
& \quad=E\left[x_{n}^{T}\left[g\left(t_{n}, x_{n}, \bar{x}_{n}\right) E\left(\Delta w_{n} \mid \mathscr{A}_{t_{n}}\right)\right]\right]=0, \\
& E\left[\left|g\left(t_{n}, x_{n}, \bar{x}_{n}\right)\right|^{2}\left|\Delta w_{n}\right|^{2}\right] \\
& \quad=E\left[\left|g\left(t_{n}, x_{n}, \bar{x}_{n}\right)\right|^{2} E\left(\left|\Delta w_{n}\right|^{2} \mid \mathscr{A}_{t_{n}}\right)\right] \\
& \quad \leq \gamma h\left(E\left|x_{n}\right|^{2}+E\left|\bar{x}_{n}\right|^{2}\right), \\
& E\left[x_{n}^{T}\left[g_{1}^{\prime}\left(t_{n}, x_{n}, \bar{x}_{n}\right) g\left(t_{n}, x_{n}, \bar{x}_{n}\right) I_{1}\right]\right] \\
& \quad=E\left[x_{n}^{T}\left[g_{1}^{\prime}\left(t_{n}, x_{n}, \bar{x}_{n}\right) g\left(t_{n}, x_{n}, \bar{x}_{n}\right) E\left(I_{1} \mid \mathscr{A}_{t_{n}}\right)\right]\right]=0, \\
& E\left[x_{n}^{T}\left[g_{2}^{\prime}\left(t_{n}, x_{n}, \bar{x}_{n}\right) g\left(t_{n}, \bar{x}_{n}, \widehat{x}_{n}\right) I_{2}\right]\right] \\
& \quad=E\left[x_{n}^{T}\left[g_{2}^{\prime}\left(t_{n}, x_{n}, \bar{x}_{n}\right) g\left(t_{n}, \bar{x}_{n}, \widehat{x}_{n}\right) E\left(I_{2} \mid \mathscr{A}_{t_{n}}\right)\right]\right]=0,
\end{aligned}
$$

and a combination of Lemma 4, (5), and (28) gives

$$
\begin{aligned}
& E\left[\left|g_{1}^{\prime}\left(t_{n}, x_{n}, \bar{x}_{n}\right)\right|^{2}\left|g\left(t_{n}, x_{n}, \bar{x}_{n}\right)\right|^{2}\left|I_{1}\right|^{2}\right] \\
& =E\left[\left|g_{1}^{\prime}\left(t_{n}, x_{n}, \bar{x}_{n}\right)\right|^{2}\left|g\left(t_{n}, x_{n}, \bar{x}_{n}\right)\right|^{2} E\left(\left|I_{1}\right|^{2} \mid \mathscr{A}_{t_{n}}\right)\right] \\
& \leq \frac{1}{2} M^{2} \gamma h\left(E\left|x_{n}\right|^{2}+E\left|\bar{x}_{n}\right|^{2}\right) \text {, } \\
& E\left[\left|g_{2}^{\prime}\left(t_{n}, x_{n}, \bar{x}_{n}\right)\right|^{2}\left|g\left(t_{n}, \bar{x}_{n}, \widehat{x}_{n}\right)\right|^{2}\left|I_{2}\right|^{2}\right] \\
& =E\left[\left|g_{2}^{\prime}\left(t_{n}, x_{n}, \bar{x}_{n}\right)\right|^{2}\left|g\left(t_{n}, \bar{x}_{n}, \widehat{x}_{n}\right)\right|^{2} E\left(\left|I_{2}\right|^{2} \mid \mathscr{A}_{t_{n}}\right)\right] \\
& \leq \frac{1}{2} N^{2} \gamma p h\left(E\left|\bar{x}_{n}\right|^{2}+E\left|\widehat{x}_{n}\right|^{2}\right) \text {. }
\end{aligned}
$$

Taking expectation on both sides of (31) and then substituting (32)-(36) into the obtained inequality yield

$$
\begin{aligned}
E\left|x_{n+1}\right|^{2} \leq & E\left|x_{n}\right|^{2}+\left(c_{1}+4 \kappa h\right) h E\left|x_{n}\right|^{2} \\
& +\left(c_{2}+4 \kappa h\right) h E\left|\bar{x}_{n}\right|^{2}+c_{3} h E\left|\widehat{x}_{n}\right|^{2} .
\end{aligned}
$$

Combining (16) and (37) derives

$$
\begin{aligned}
E\left|x_{n+1}\right|^{2} \leq & E\left|x_{n}\right|^{2}+\left(c_{1}+4 \kappa h\right) h E\left|x_{n}\right|^{2} \\
& +\left(c_{2}+4 \kappa h\right) h \delta_{n} E\left|x_{n-v_{n}+1}\right|^{2} \\
& +\left(c_{2}+4 \kappa h\right) \times h\left(1-\delta_{n}\right) E\left|x_{n-v_{n}}\right|^{2} \\
& +c_{3} h \bar{\delta}_{n} E\left|x_{n-\bar{v}_{n}+1}\right|^{2}+c_{3} h\left(1-\bar{\delta}_{n}\right) E\left|x_{n-\bar{v}_{n}}\right|^{2} .
\end{aligned}
$$

An induction to (38) yields

$$
\begin{aligned}
E\left|x_{n+1}\right|^{2} \leq & E|\xi|^{2}+\left(c_{1}+4 \kappa h\right) h \sum_{i=1}^{n} E\left|x_{i}\right|^{2} \\
& +\left(c_{2}+4 \kappa h\right) h \sum_{i=1}^{n} \delta_{i} E\left|x_{i-v_{i}+1}\right|^{2} \\
& +\left(c_{2}+4 \kappa h\right) h \times \sum_{i=1}^{n}\left(1-\delta_{i}\right) E\left|x_{i-v_{i}}\right|^{2} \\
& +c_{3} h \sum_{i=1}^{n} \bar{\delta}_{i} E\left|x_{i-\bar{v}_{i}+1}\right|^{2}+c_{3} h \sum_{i=1}^{n}\left(1-\bar{\delta}_{i}\right) E\left|x_{i-\bar{\nu}_{i}}\right|^{2} .
\end{aligned}
$$

Applying Lemma 5 to (39), it follows that

$$
\begin{aligned}
E\left|x_{n+1}\right|^{2} \leq & E|\xi|^{2}+\left(c_{1}+4 \kappa h\right) h \sum_{i=1}^{n} E\left|x_{i}\right|^{2} \\
& +\left(c_{2}+4 \kappa h\right)(r+1) h \sum_{i=1}^{n} E\left|x_{i}\right|^{2} \\
& +\left(c_{2}+4 \kappa h\right)(r+1) h \sum_{i=0}^{n} E\left|x_{i}\right|^{2} \\
& +c_{3}(r+1) h \sum_{i=1}^{n} E\left|x_{i}\right|^{2}+c_{3} h(r+1) \sum_{i=0}^{n} E\left|x_{i}\right|^{2}
\end{aligned}
$$




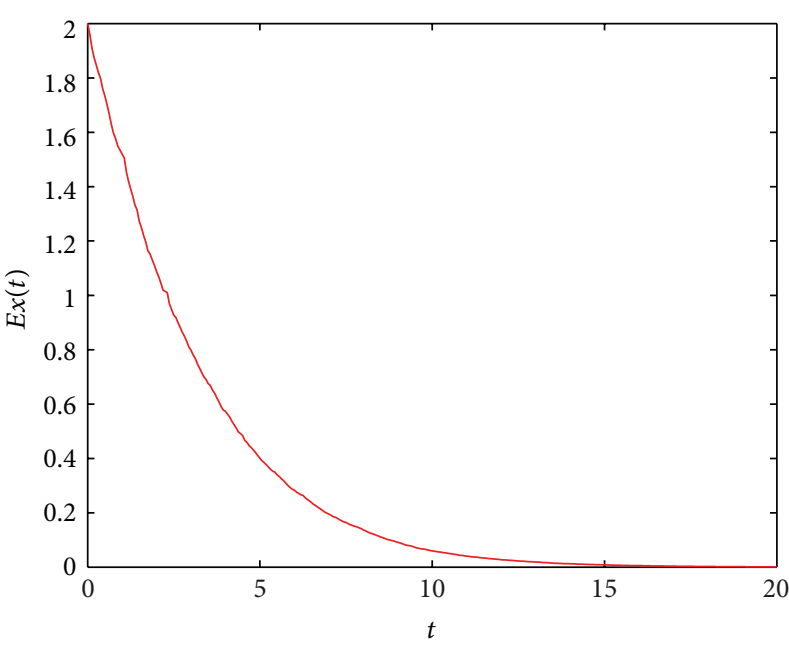

$-h=1 / 2^{4}$

(a)

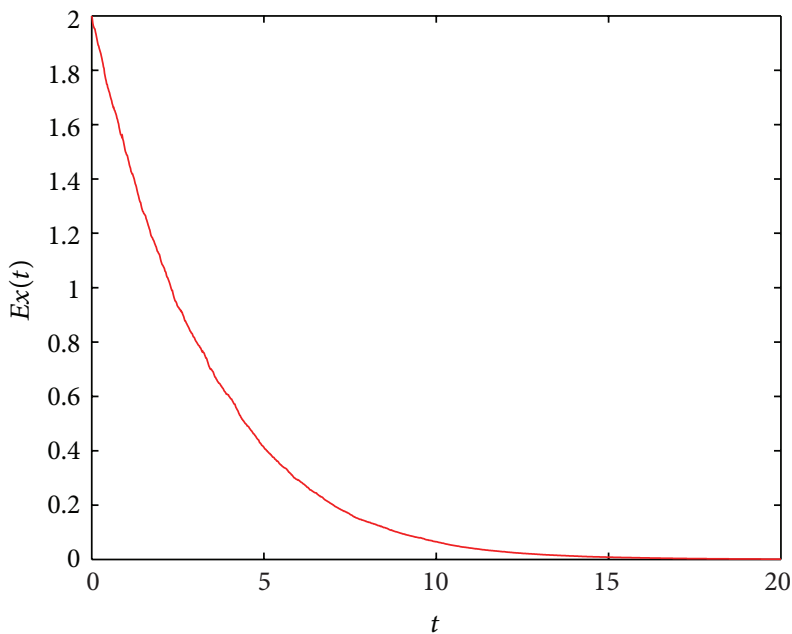

$h=1 / 2^{6}$

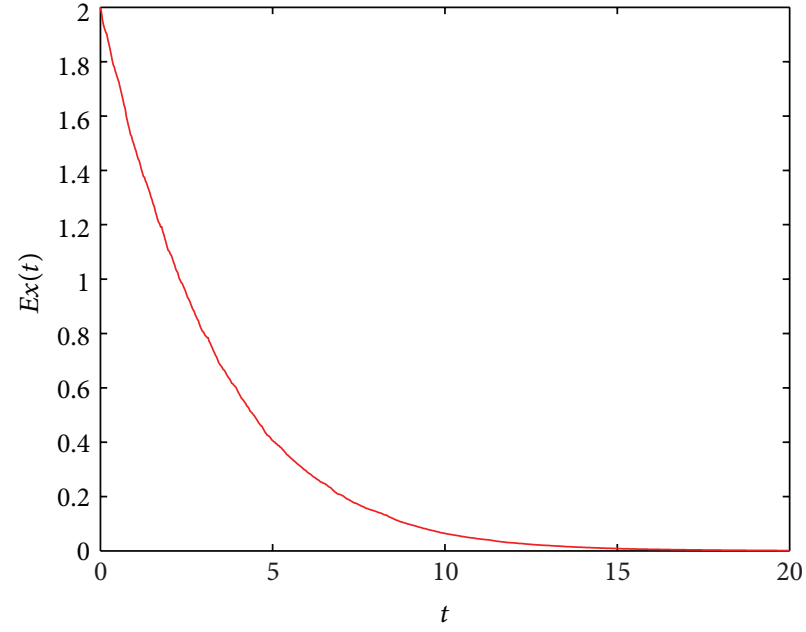

$-h=1 / 2^{5}$

(b)

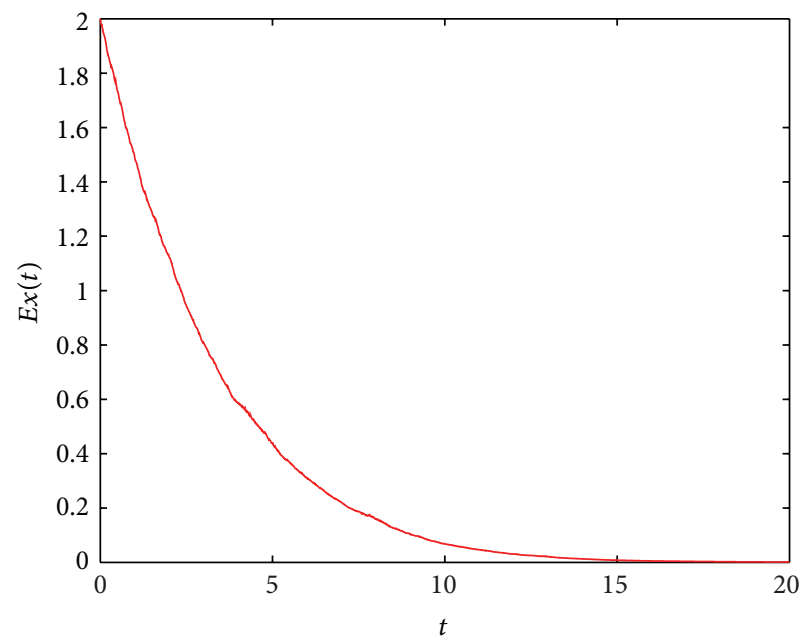

$h=1 / 2^{7}$

(c)

(d)

FIgURE 1: Numerical solutions with stepsizes $h=1 / 2^{4}, 1 / 2^{5}, 1 / 2^{6}, 1 / 2^{7}$.

$$
\begin{gathered}
\leq\left[1+\left(c_{2}+4 \kappa\right)(r+1)+c_{3}(r+1)\right] E|\xi|^{2} \\
+h\left[c_{1}+2 c_{2}(r+1)+2 c_{3}(r+1)+4 \kappa h\right. \\
+8 \kappa(r+1) h] \sum_{i=1}^{n} E\left|x_{i}\right|^{2} .
\end{gathered}
$$

This shows that the positive series $\sum_{i=1}^{n} E\left|x_{i}\right|^{2}$ is bounded when (29) holds and $h \in\left(0, h_{0}\right)$, where

$$
h_{0}=\min \left\{1, \frac{-c_{1}-2 c_{2}(r+1)-2 c_{3}(r+1)}{4 \kappa[1+2(r+1)]}\right\} \text {. }
$$

Therefore, it holds that $\lim _{n \rightarrow \infty} E\left|x_{n}\right|^{2}=0$. This completes the proof.

\section{Numerical Illustration}

In this section, we give a numerical example to illustrate the obtained theoretical results. Consider the following stochastic pantograph equation:

$$
\begin{gathered}
d x(t)=-\frac{1}{4} x(t)\left[1+\cos ^{2} x\left(\frac{t}{2}\right)\right] d t \\
+\frac{1}{5} x(t) x\left(\frac{t}{2}\right) d w(t), \quad t>0, \\
x(0)=2 .
\end{gathered}
$$

It is easy to verify that the conditions of Theorems 2 and 6 can be satisfied with parameters

$$
\alpha=\frac{1}{4}, \quad \beta=0, \quad \gamma=\frac{1}{50}, \quad \kappa=\frac{1}{16},
$$




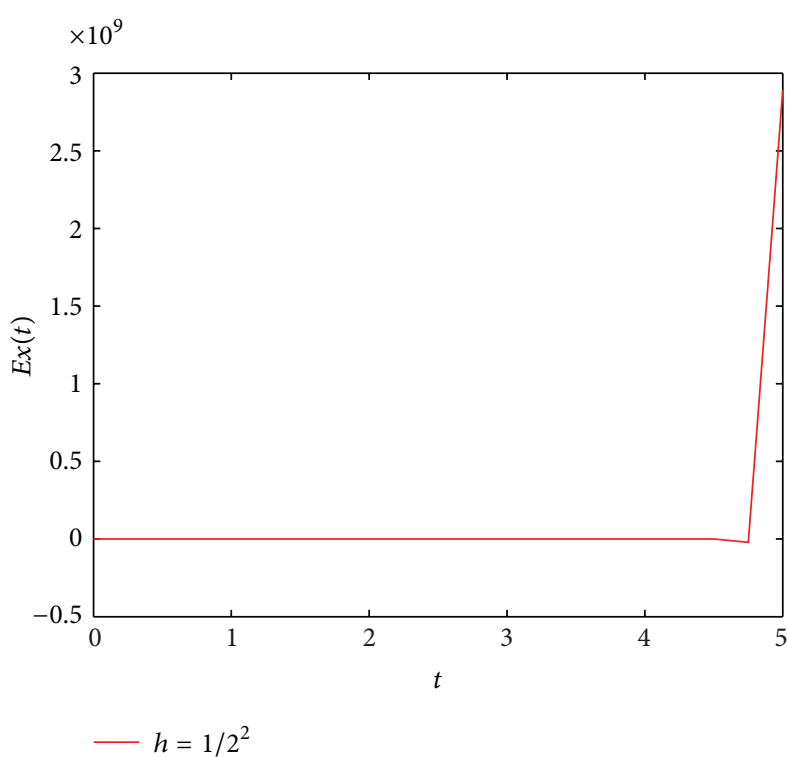

(a)

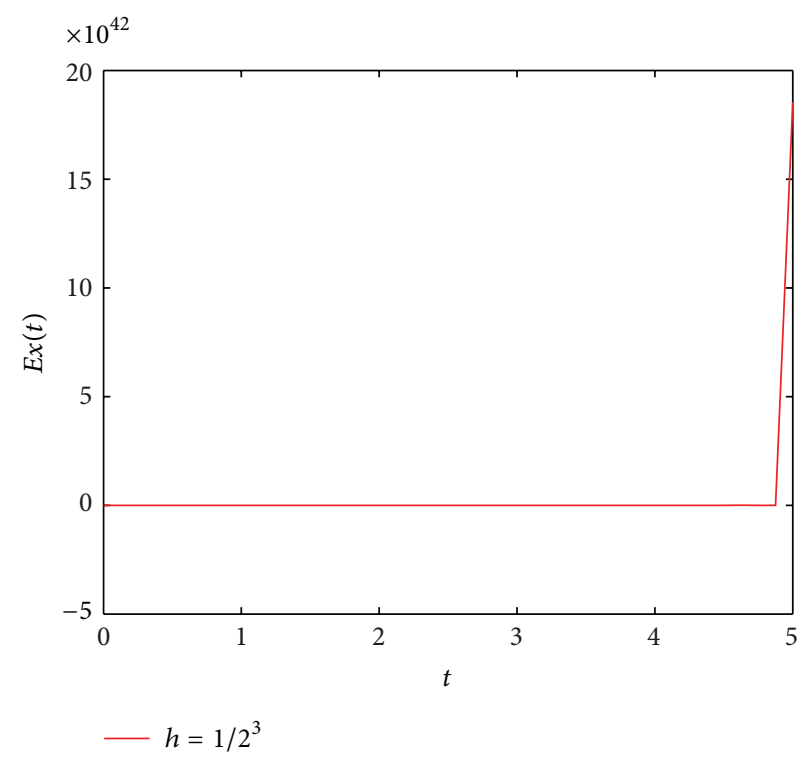

(b)

FIgURE 2: Numerical solutions with stepsizes $h=1 / 2^{2}, 1 / 2^{3}$.

$$
r=1, \quad M=N=\frac{1}{5}, \quad h_{0}=\frac{428}{6250}>\frac{1}{2^{4}} .
$$

Hence, both the solution of (42) and its solving method (14) are all MS-stable.

Applying the extended Milstein method (14)-(16), with stepsizes $h=1 / 2^{4}, 1 / 2^{5}, 1 / 2^{6}, 1 / 2^{7} \in\left(0, h_{0}\right]$, respectively, to (42) on interval $[0,20]$, we can obtain four groups of numerical solutions (see Figure 1), where we take the average of 1000 block samples. Figure 1 shows that the numerical solutions are all stable. However, if we take a larger stepsize, then the numerical stability cannot be assured. This is shown in Figure 2, where stepsizes $h=1 / 2^{2}, 1 / 2^{3} \notin\left(0, h_{0}\right]$ are used, which leads to two groups of unstable solutions.

\section{Conclusions}

In this paper, a class of extended Milstein methods for solving nonlinear stochastic pantograph equations are suggested. A mean-square stability criterion for this type of equations is presented. It is proved that, under the suitable conditions, if the stepsize satisfies the sufficient condition $h \leq h_{0}$, where $h_{0}$ is given by (41), then the Milstein methods preserve the mean-square stability. How does one obtain an exact critical stepsize $\widetilde{h}_{0}$ such that the method is stable for $h \in\left(0, \widetilde{h}_{0}\right]$ and unstable for $h \in\left(\widetilde{h}_{0},+\infty\right)$ ? This is a difficult problem which keeps open at present. We will work on it in the future research.

\section{Acknowledgments}

This work is supported by NSFC (nos. 11171125, 91130003, 11201162, and 11301099), NSFH (no. 2011CDB289), Humanities and Social Science Research Projects in Ministry of
Education (13YJA910003), the Doctor Scientific Research Foundation of Guangxi Normal University, and the Fundamental Research Funds for the Central Universities, HUST (2011QN168).

\section{References}

[1] X. Mao, Stochastic Differential Equations and Their Applications, Horwood Publishing Limited, Chichester, UK, 1997.

[2] X. Mao and S. Sabanis, "Numerical solutions of stochastic differential delay equations under local Lipschitz condition," Journal of Computational and Applied Mathematics, vol. 151, no. 1, pp. 215-227, 2003.

[3] C. T. H. Baker and E. Buckwar, "Numerical analysis of explicit one-step methods for stochastic delay differential equations," LMS Journal of Computation and Mathematics, vol. 3, pp. 315335, 2000.

[4] E. Buckwar, "Introduction to the numerical analysis of stochastic delay differential equations," Journal of Computational and Applied Mathematics, vol. 125, no. 1-2, pp. 297-307, 2000.

[5] E. Buckwar, "One-step approximations for stochastic functional differential equations," Applied Numerical Mathematics, vol. 56, no. 5, pp. 667-681, 2006.

[6] U. Küchler and E. Platen, "Strong discrete time approximation of stochastic differential equations with time delay," Mathematics and Computers in Simulation, vol. 54, no. 1-3, pp. 189-205, 2000.

[7] W. Cao, M. Liu, and Z. Fan, "MS-stability of the Euler-Maruyama method for stochastic differential delay equations," Applied Mathematics and Computation, vol. 159, no. 1, pp. 127-135, 2004.

[8] M. Liu, W. Cao, and Z. Fan, "Convergence and stability of the semi-implicit Euler method for a linear stochastic differential delay equation," Journal of Computational and Applied Mathematics, vol. 170, no. 2, pp. 255-268, 2004. 
[9] Z. Wang and C. Zhang, "An analysis of stability of Milstein method for stochastic differential equations with delay," Computers \& Mathematics with Applications, vol. 51, no. 9-10, pp. 1445-1452, 2006.

[10] C. T. H. Baker and E. Buckwar, "Exponential stability in $p$ th mean of solutions, and of convergent Euler-type solutions, of stochastic delay differential equations," Journal of Computational and Applied Mathematics, vol. 184, no. 2, pp. 404-427, 2005.

[11] Z. Y. Wang and C. J. Zhang, "Mean-square stability of the Milstein method for solving nonlinear stochastic delay differential equations," Mathematica Applicata, vol. 21, no. 1, pp. 201-206, 2008.

[12] C. Zhang and G. Sun, "The discrete dynamics of nonlinear infinite-delay-differential equations," Applied Mathematics Letters, vol. 15, no. 5, pp. 521-526, 2002.

[13] C. Zhang and G. Sun, "Nonlinear stability of Runge-Kutta methods applied to infinite-delay-differential equations," Mathematical and Computer Modelling, vol. 39, no. 4-5, pp. 495-503, 2004.

[14] Z.-C. Fan and M.-Z. Liu, "The asymptotically mean square stability of the linear stochastic pantograph equation," Mathematica Applicata, vol. 20, no. 3, pp. 519-523, 2007.

[15] Z. Fan, M. Song, and M. Liu, "The $\alpha$ th moment stability for the stochastic pantograph equation," Journal of Computational and Applied Mathematics, vol. 233, no. 2, pp. 109-120, 2009.

[16] Y. Xiao and H. Zhang, "Convergence and stability of numerical methods with variable step size for stochastic pantograph differential equations," International Journal of Computer Mathematics, vol. 88, no. 14, pp. 2955-2968, 2011.

[17] Y. Xiao, M. Song, and M. Liu, "Convergence and stability of the semi-implicit Euler method with variable stepsize for a linear stochastic pantograph differential equation," International Journal of Numerical Analysis and Modeling, vol. 8, no. 2, pp. 214225, 2011. 


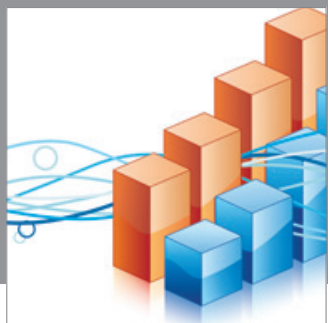

Advances in

Operations Research

mansans

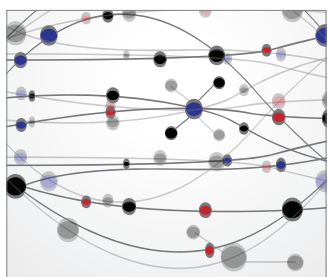

The Scientific World Journal
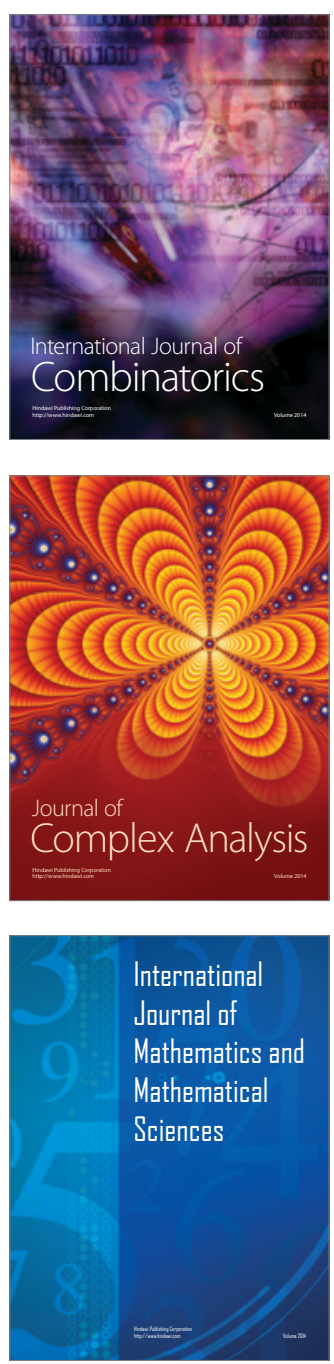
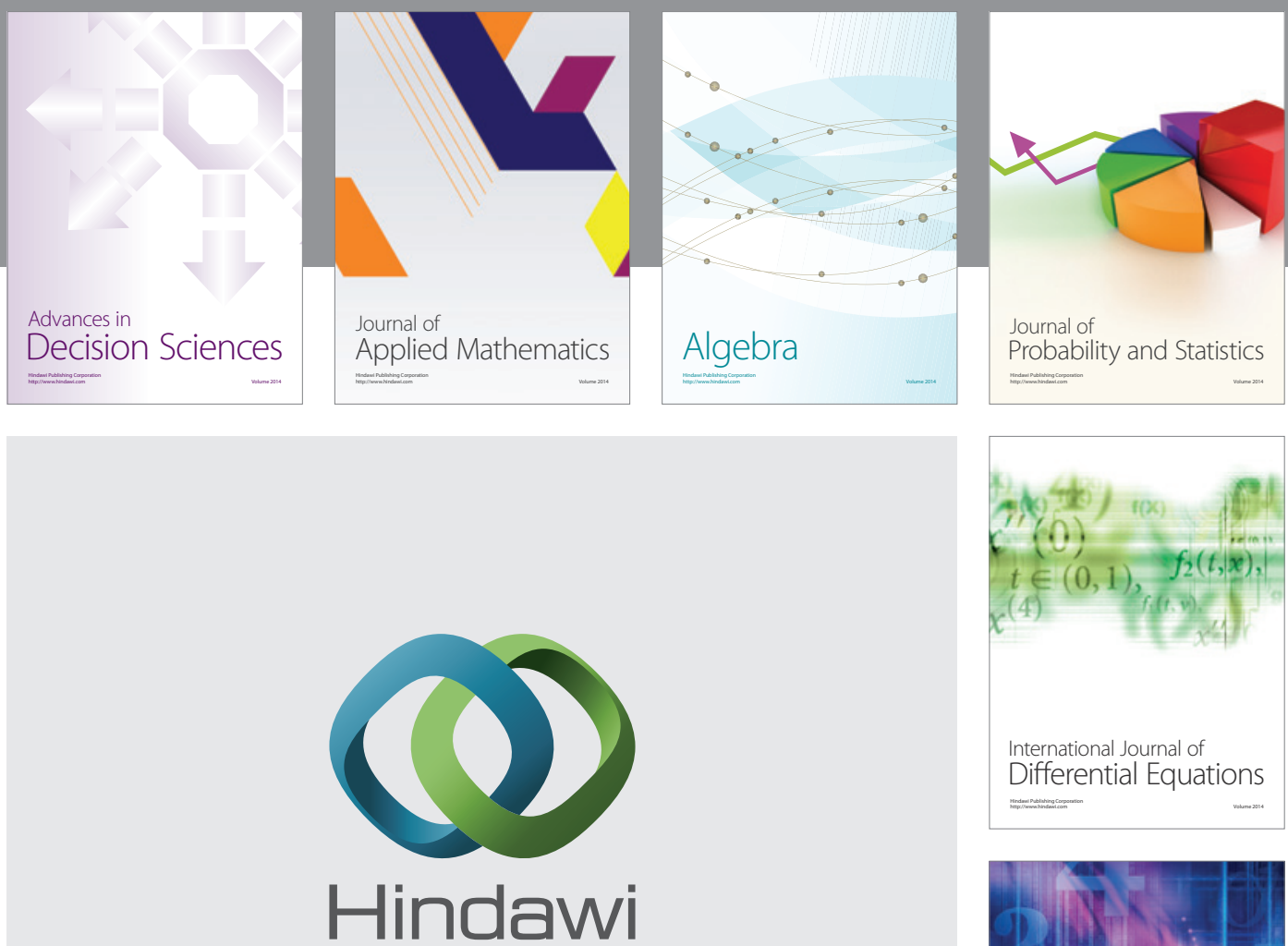

Submit your manuscripts at http://www.hindawi.com
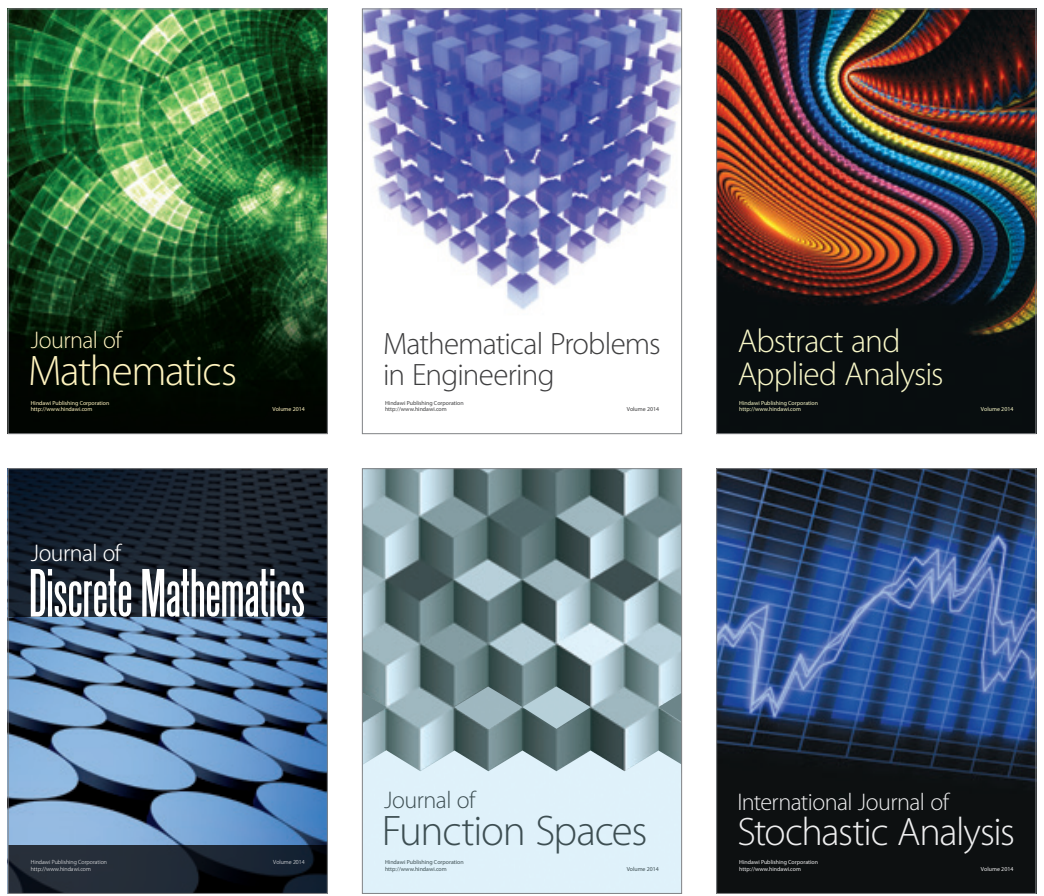

Journal of

Function Spaces

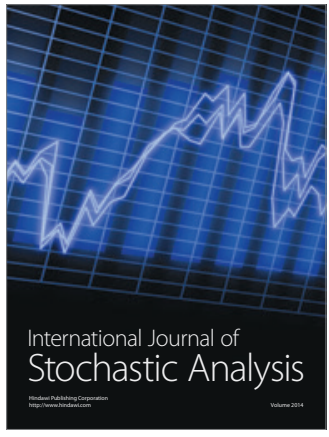

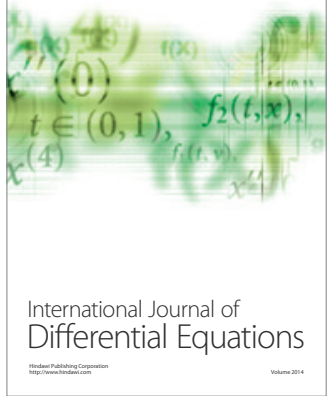
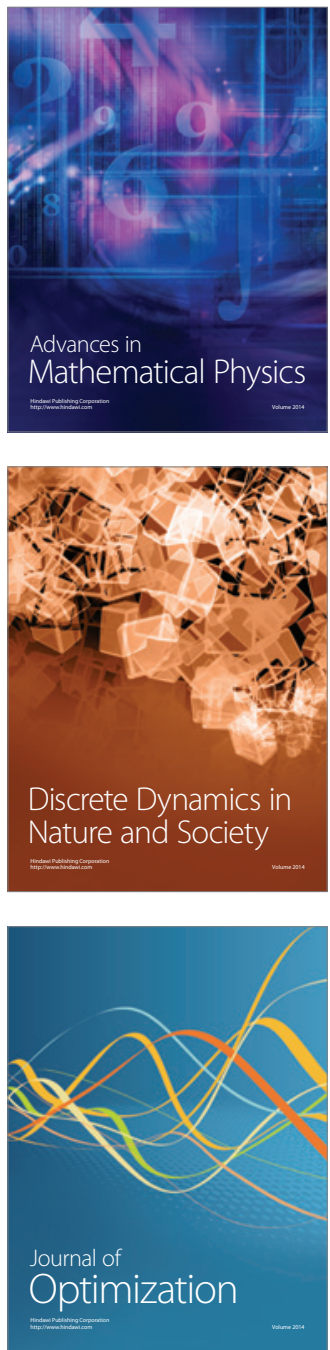